\title{
Política hemisférica de los noventa: elementos del debate estratégico en los Estados Unidos
}

\author{
José A. Morandé L.
}

Este artículo analiza primeramente los alcances generales del cambio de la política hemisférica de los Estados Unidos en la década de los noventa. El reemplazo de la estrategia de la "contención" por la nueva política norteamericana de la "ampliación", ha significado la reafirmación por parte de ese país de sus intereses estratégicos tradicionales en el hemisferio, junto a una adhesión y compromiso mayoritario del continente americano por los principios de la democracia y la economía de mercado. No obstante este creciente consenso interamericano, los nuevos desafíos y oportunidades que ofrece la actual agenda mundial y regional han generado un debate intelectual y político en los círculos norteamericanistas del país del Norte. Para esto, entonces, a partir de las visiones mundiales realista y liberal elaboradas por Barry Hughes, este ensayo intenta una explicación de la política exterior de Estados Unidos en la región sobre la base de la interpretación de su contenido por parte de especialistas y políticos norteamericanos. Por medio de la utilización de dos categorías amplias (realismo-cauteloso y liberalismo-optimista), el presente trabajo intenta agrupar y explicar algunas de las tendencias que se manifiestan en Estados Unidos. Ambas categorías, si bien son coincidentes en cuanto a materias y valores a promover en la región, difieren sin embargo respecto a prescripciones, instrumentos de política exteriory, por último, a la prioridad que el continente tendría en la política hemisférica norteamericana.

\section{La transformación del escenario hemisférico.}

Durante un extenso período de la postguerra, las relaciones hemisféricas fueron concebidas por los Estados Unidos en el marco de una estrategia 
global, cuya coherencia ideológica permitió una articulación regional de sus objetivos de seguridad, políticos y económicos.

Más específicamente, la política hemisférica de la Guerra Fría definida como de la "contención" centró su preocupación en la protección de la integridad continental contra la amenaza militar soviética y en la mantención de un acceso seguro a las materias primas de la región. Por su parte, los objetivos y tareas para el desarrollo político y económico en el hemisferio occidental fueron genëralmente subordinados a los intereses estadounidenses de seguridad.

En lo que se refiere al proceso de formulación de la política exterior de la contención, esta visión global identificó prioritariamente un interés nacional de carácter geopolítico en el que se reconoció un papel preeminente y decisivo al Estado norteamericano. En otras palabras, las decisiones fueron adoptadas por los funcionarios oficiales para alcanzar y optimizar aquellos objetivos domésticos e internacionales que apoyaban la estrategia global, superponiéndose al mismo tiempo a las presiones internas y foráneas de los grupos de interés.

En términos de la agenda hemisférica, ésta fue organizada y estructurada bajo el principio orientador de la lucha Este-Oeste. Mas, a pesar de la rigidez ideológica de la Guerra Fría, los instrumentos de política exterior escogidos por diferentes gobiernos norteamericanos respondieron a una combinación entre visiones realistas e idealistas. Esto fue cierto en la medida en que estos instrumentos de política exterior incluyeron simultáneamente programas tan diversos como de asistencia militar y antisubversivos o de asistencia al desarrollo, ayuda externa y la Alianza para el Progreso, entre otros. ${ }^{1}$

Bajo tales circunstancias, la agenda regional fue dividida y debatida entre materias y prioridades enfatizadas por las administraciones estadounidenses de acuerdo con el desarrollo dialéctico de la relación EsteOeste. Al mismo tiempo, la política exterior de los Estados Unidos fue diseñada en forma desconectada e inmune al debate valórico que entonces preocupaba a la política doméstica. ${ }^{2}$

Sin embargo, las recientes transformaciones del sistema intemacional contemporáneo -mayor interdependencia entre diversos actores estata-

\footnotetext{
"Mark Falcoff, "A look at Latin America", en: Nicholas X. Rizopoulos (ed.), Sea-Changes: American Foreign Policy in a World Transformed, (New York: Council on Foreign Relations Press, 1989), pp. 72-73.

${ }^{2}$ Claude Pomerleam, "Morality and U.S. Foreign Policy in a Pos-Cold War World", trabajo presentado en la Joint Annual Meeting de International Studies Association/South and International Security Studies, 15-17 de octubre, 1993.
} 
les y no-gubernamentales y la desaparición de la amenaza soviéticahan contribuido a cambiar el alcance y contenido de la política exterior de Estados Unidos. Desde el momento en que no existe mayor amenaza a la seguridad e intereses norteamericanos en el hemisferio, emerge una agenda continental más consensual entre políticos y especialistas estadounidenses. Esta tendencia ha sido reforzada con el compromiso creciente de los líderes de los gobiernos del hemisferio con la democracia, reforma y libertad económica.

No obstante estas coincidencias, en el debate actual sobre un nuevo orden internacional persisten en los Estados Unidos algunas percepciones y visiones diferentes respecto de un papel renovado del país del Norte en la región: El fin de la Guerra Fria ha sido evaluado diferentemente por latinoamericanistas del mundo académico y político norteamericano en dos tendencias contrapuestas, es decir, como una oportunidad para incrementar o disminuir una asociación continental. Ambas visiones, esto es, una mayor o menor aproximación regional, dependen en gran parte de los supuestos tradicionales de los paradigmas hemisféricos de la política exterior norteamericana. Con todo, esta discusión es menos antagónica que en el pasado, puesto que hoy día existe mayor congruencia de valores en el escenario internacional.

Una reciente formulación de la política exterior norteamericana que reemplazó a la vieja estrategia de la contención es la política denominada de la "ampliación". Esta combina la promoción de la democracia y la economía de mercado con los intereses estratégicos tradicionales de Estados Unidos. A pesar de su pragmatismo, la estrategia de la ampliación reconoce que la democracia y la economía de mercado no son los únicos intereses que mueven al país del Norte, ya que todavia es posible para éste colaborar y defender a naciones no-democráticas si se trata de alcanzar beneficios mutuos. ${ }^{3}$

Es más, aun cuando valores como los mencionados anteriormente forman parte de la cultura y de la política exterior de los Estados Unidos, ellos no son siempre percibidos como prácticos y coincidentes con el interés nacional. En efecto, si la promoción de elecciones democráticas en el exterior desencadenaran movimientos populares antinorteamericanos, entonces la promoción de la democracia podría ser contraproducenté para los intereses de Estados Unidos. ${ }^{4}$

\footnotetext{
${ }^{3}$ Anthony Lake, "Estados Unidos y su presencia exterior", Politica Exterior, N\$35, VII, otoño 1993, pp. 116-117.

${ }^{4}$ Kim R. Holmes, 'Defining National Security and American Interests", en: Kim R Holmes (ed.), A Safe and Prosperous America: A U.S. Foreign and Defense Policy Blueprint, (Washington, D.C.: The Heritage Foundation, junio 1994), pp. 5-6.
} 
En otras palabras, pese al creciente consenso estratégico de las élites norteamericanas en lo que dice relación a los asuntos globales del mundo de la post-Guerra Fría, se puede inferir también que se estaría gestando al mismo tiempo un nuevo debate intelectual y político en el pais del Norte frente a los desafíos y oportunidades régionales que representa la actual estrategia.

A partir del escenario hemisférico actual y en relación al temä de este trabajo, interesa entonces indagar hasta qué punto se presentan interpretaciones y visiones diferentes en círculos norteamericanos respecto á las relaciones hemisféricas contemporáneas.

A modo de explorar algunas respuestas tentativas a esta interrogante, recurriremos en parte a los aportes conceptuales y analíticos que entrega el enfoque comparado de "visión mundial " elaborado por Hughes. 5 Este enfoque ofrece un marco explicativo amplio en el que se combinan diferentes perspectivas y unidades de análisis con el elemento prescriptivo de los valores. De esta forma se podrá intentar una explicación de la estrategia actual de política exterior de Estados Unidos en la región, tanto en su contenido como en su interpretación por parte de especialistas y políticos norteamericanos.

\section{Visiones y opciones en las relaciones hemisféricas.}

Con el fin de la estrategia global de la contención y en el contexto de las actuales relaciones interamericanas es posible advertir un nuevo debate entre conservadores y liberales norteamericanos. En efecto, la nueva estrategia de la ampliación ha originado una discusión renovada acerca del papel de Estados Unidos en el hemisferio occidental y de sus vínculos deseables con los países que lo integran.

Ya a fines de la década de 1980, algunos políticos y especialistas estadounidenses pensaron que la región debería permanecer marginada en la estructuración del emergente orden mundial. Otros, en cambio, consideraron que la desaparición de la Guerra Fría sería una buena oportunidad para renovar con nuevos bríos la cooperación hemisférica.

Los que adhieren al primer grupo - realistas - subrayaron que una menor preocupación de Estados Unidos en la región era una expresión normal y adecuada a los nuevos tiempos, por cuanto existirían limitadas posibilidades de amenazas regionales a los intereses de seguridad nor-

\footnotetext{
${ }_{5}^{5}$ Barry B. Hughes, Continutity and Change in World Politics, (Englewood Cliffs, N.J.: Prentice-Hall, 1994), segunda edición, pp. 41-42.
} 
teamericanos. Los liberales, por su parte, identificados estrechamente con la cooperación internacional, afirmaron en ese entonces que Estados Unidos debería poner mucho más atención en el continente ya que el objetivo de una deseada asociación mutua fue derrotado por las políticas de la Guerra Fría. ${ }^{6}$

Durante los noventa, sin embargo, este debate ha estado más concentrado en los principios, propósitos e instrumentos de política exterior que contempla la estrategia de la ampliación. La promoción de la democracia y economía de mercado es en el presente un consenso hemisférico creciente. Ambos valores se apoyan y propagan a través de los vínculos transnacionales e interdependientes que también influyen y se manifiestan en las naciones y conductas de los líderes de la región. Por lo tanto, las convergencias y disensos entre realistas y liberales son ideológicamente menos agudos y sesgados que durante la vigencia de la estrategia de la contención.

Aun cuando el ambiente de la post-Guerra Fría es más consensual, tanto los realistas como los liberales intentan explicar y prescribir las relaciones hemisféricas de acuerdo a sus supuestos y valores básicos.

El realismo, como una visión del mundo en la concepción de Hughes, enfatiza valores y prescripciones. Desde que la seguridad nacional y el propósito de autonomía son los valores dominantes de los Estados, entonces los realistas justifican el uso del poder (aun la fuerza) para defenderse a sí mismos y la preservación del sistema de Estados. ${ }^{7}$ No obstante, las versiones más modernas del realismo han ampliado la participación de actores tradicionales en el escenario mundial como la incorporación de nuevos temas en la agenda internacional.

En otras palabras, el neo-realismo está preocupado también con las nuevas bases del cambio contemporáneo en el sistema internacional. Así, en términos de tópicos y prescripciones, esta visión mundial considera en la actualidad, entre otros, la amenaza global de las armas nucleares, el poder cambiante de los Estados Unidos, el libre comercio en el mundo y el impacto del terrorismo internacional, para lo cual promueve ahora activamente la cooperación interestatal.

Como visión mundial, el liberalismo ve al sistema global en el marco de una estructura en la cual las comunidades - tanto locales como globales - se transforman en el foco de análisis. Estos valores son prescriptivos si contribuyen a configurar políticas y si también ayudan a

\footnotetext{
"Joseph S. Tulchin "Estados Unidos y América Latina en el mundo", en: Francisco Rojas y William C. Smith (eds.), El Cono Sur y las transformaciones globales, (Santiago: FLACSO/North South Center/CLADE, 1994), pp. 153-154.

${ }^{7}$ Véase Barry B. Hughes, op. cit., pp. 52-53.
} 
determinar actitudes futuras y a garantizar las reglas y procedimientos de la conducta internacional.

El carácter globalista e ideálista de esta visión mundial permite considerar a las organizaciones internacionales como los actores esenciales de la política mundial. Asimismo, estas organizaciones contribuyen a que las comunidades humanas conjuntamente con las naciones-Estados mantengan el orden, ayuden a una política más redistributiva de recursos y oportunidades en el mundo, y con ello a prosperar en relativa seguridad.

En términos de las relaciones hemisféricas y de acuerdo a las visiones tradicionales norteamericanas en este campo, la pregunta que surge es cómo trazar la línea diferenciadora entre los realistas y liberales estadounidenses en la era de la post-Guerra Fría.

Para responder a esta interrogante se intentará agrupar algunas de las tendencias hemisféricas actuales en el marco de dos amplias cateǵorías: Realismo-Cauteloso y Liberalismo-Optimista. ${ }^{8}$

\section{Realismo-Cauteloso.}

Las percepciones y visiones de esta categoría enfatizan que la región pierde interés para los Estados Unidos después de la era de la Guerra Fría. Este es un proceso que se inició con el retiro gradual de la influencia soviética en el hemisferio y finalizó desde el momento en que la amenaza soviética desapareció completamente en el continente.

La lógica de este desacople norteamericano del hemisferio no es sólo geoestratégica sino que también económica. Es decir, el retiro es refor-

\footnotetext{
${ }_{8}^{8}$ Para la categoría Realismo-Cauteloso, véase Mark Falcoff, "A Look at Latin America", op. cit., y también, "Desafios a la seguridad en el hemisferio: Una visión norteamericana", en: Heraldo Muñoz (ed.), El fin del fantasma: las relaciones interamericanas después de la Guerra Fria, (Santiago: Hachette, 1992). Howard J. Wiarda, "U.S. Strategic Policy in Latin America in the Pos-Cold War Era", en: L. Erik Kjonnerod (ed.), Evolving U.S. Strategy for Latin America and the Caribbean, (Washington, D.C.: National Defense University Press, 1992). Del mismo autor, "El avance de la democracia en América Latina", Ciencia Politica, N²6, Primer Trimestre, 1992.

En la categoria Liberalismo-Optimista, véase Richard Feinberg, "Substantive Symmetry in Hemispheric Relations", Dispatch, N'14, Vol.5, marzo 1994. Robert Pastor, "The Latin American Option", Foreign Policy, No8, Fall 1992; y "Estados Unidos y América Latina en los noventa", Ciencia Palitica, No30, Primer Trimestre, 1993. Thomas Carothers, "The United States and Latin America After the Cold War", Working Papers, N'184, Washington, D.C., The Wilson Center, Latin American Program, 1990. Peter Hakim, "NAFTA and After: A New Era for the United States and Latin America?", Current History, Vol. 93, marzo 1994. Abraham F. Lowenthal, "Rediscovering Latin America", Foreign Affairs, Vol. 69, Fall 1990. Del mismo autor véase también "Latin America: Ready for Partnership?", Foreign Affairs, Vol. 72, 1993; y "U.S. Policy Toward Latin America", en: Robert J. Art y Seyom Brown (eds.), U.S. Foreign Policy: The Search for a New Role, (New York: Macmillan Publishing Company, 1993).
} 
zado por las presiones domésticas de Estados Unidos orientadas a priorizar los temas de su déficit presupuestario y a mejorar su competitividad en la economía internacional. De esta forma se explica también los muy escasos recursos de ayuda externa que hoy día se disponen para un mayor compromiso norteamericano en el hemisferio. ${ }^{9}$

Por otra parte, esta perspectiva realista presenta algunas dudas acerca del éxito de las políticas macroeconómicas implementadas en la región si no hay un real compromiso por parte de los gobiernos del hemisferio con las reformas de libre mercado.

A través de estas políticas —de acuerdo a esta misma visión- es posible alcanzar crecimiento económico regional, generar confianza empresarial, atraer capital externo, mantener el capital local en casa, y más importante, proveer nuevos mercados para los Estados Unidos en la región. 10

En lo que dice relación con los instrumentos de política exterior hemisférica, algunos realistas sospechan de los enfoques multilaterales para abordar los temas regionales. En otras palabras, para ellos las relaciones bilaterales son más viables para defender los intereses nacionales del país del Norte.

Un caso concreto que avala esta posición fue la reacción del ex-Secretario Adjunto para los Asuntos Interamericanos quien afirmó que el Presidente Clinton sacrificó el interés nacional de Estados Unidos en Haití para satisfacer los intereses personales del presidente de este país, Aristide. Al mismo tiempo - agrega el funcionario norteamericanolos Estados Unidos aceptaron la presión latinoamericana de tomar este caso como un ejemplo del nuevo consenso de la OEA acerca de las sanciones regionales contra los golpes militares. ${ }^{11}$

Finalmente, para esta perspectiva también existen problemas transnacionales en las relaciones hemisféricas tales como narcotráfico, amenazas ecológicas, inmigraciones, comupción y violencia. Estos problemas generan inestabilidad política y social en la región cuyos efectos negativos podrían resultar en un menor interés de los Estados Unidos en el hemisferio, especialmente en lo que respecta a las percepciones y actitudes del público norteamericano. ${ }^{12}$

\footnotetext{
${ }^{9}$ Howard J. Wiarda, "U.S. Strategic Policy in Latin America in the Pos-Cold War Era", en: L. Erik Kjonnerod (ed.), op. cit., p. 24.

${ }^{10}$ Mark Falcoff, "A look at Latin America", op. cit., p. 81.

${ }^{11}$ Elliot Abrahams, “¿Por qué Aristide?”, El Mercurio, Santiago, Chile, 28 de octubre, 1993, p. A-2,

${ }^{12}$ Véase H. Wiarda, op. cit., pp. 24-25.
} 
En síntesis, esta visión que enfatiza por sobre todo la defensa del interés nacional, ve un papel reducido de los Estados Unidos en el hemisferio después de la Guerra Fría. Sin embargo, para los realistas, los desafios verdaderos que confronta el país del Norte en el futuro están relacionados con el desarrollo político y económico de la región. Por medio de la promoción de valores como la democracia y la economía de mercado, este desarrollo -el cual se inscribe en los intereses de largo alcance norteamericanos-podría ser alcanzado. Con este logro, la gente no se sentiría presionada a emigrar, a cultivar y traficar drogas, y a destruir la naturaleza y su ecosistema. ${ }^{13}$

\section{Liberalismo-Optimista.}

Esta categoría incorpora la visión que sostiene que el fin de la Guerra Fría es una buena oportunidad para incrementar y mejorar las relaciones hemisféricas debido a que la región está fuertemente entrelazada con los Estados Unidos.

Desde que el anticomunismo ya no es más la preocupación dominante en el hemisferio occidental, existen argumentos y estímulos muy positivos para que la potencia norteamericana se mantenga presente en la región. En primer lugar, el desarrollo democrático como una preocupación política mutua y una mayor convergencia en valores regionales. El avance del crecimiento económico a través de un modelo de orientación de libre mercado es otra coincidencia continental a incrementar en un marco de cooperación multilateral.

Así, la inversión norteamericana prosperará si el hemisferio prospera. El comercio regional también es importante, ya que un mejoramiento del desarrollo económico del área incrementará el empleo en el país del Norte. ${ }^{14}$

La combinación de reformas de mercado y el renovado crecimiento regional es considerado claramente como un factor positivo para la economía norteamericana. En efecto, la relación comercial de ésta con el hemisferio -incluyendo Canadá- hace del continente el más importante socio comercial de los Estados Unidos con exportaciones norteamericanas de 166 billones de dólares aproximadamente en 1992.15

\footnotetext{
${ }^{13}$ Véase Mark Falcoff, op. cit., pp. 79-80.

14 Thomas Carothers, "The United States and Latin America After the Cold War", op. cit., pp. 9-10.

${ }^{15}$ Alexander F. Watson, "U.S. Latin America Relations in the 1990's: 'Toward a Mature Partnership", Dispatch, Vol. 5, No11, March 14, 1994, p. 154.
} 
Otro impacto adicional importante de la región en los Estados Unidos proviene de la migración masiva hacia éste, especialmente de México, el Caribe y Centroamérica. Los aspectos negativos de este vínculo estructural tendrán que ser resueltos - de acuerdo a los liberales-con una fuerte cooperación entre las naciones afectadas del continente. ${ }^{16} \mathrm{La}$ misma cooperación es necesaria para abordar el problema de los narcóticos, la protección ambiental, el desarrollo y manejo de recursos, el combate al terrorismo, la prevención de enfermedades mortales como el SIDA y el control de la proliferación nuclear.

Culturalmente, para esta perspectiva los Estados Unidos tienen un fuerte compromiso con el estricto respeto por los derechos humanos y derechos individuales en el hemisferio. Si la región se encamina decididamente hacia regímenes democráticos, entonces será posible alcanzar una creciente convergencia en el apoyo a la causa de los derechos humanos.

El multilateralismo regional es promovido por los liberales como una herramienta política importante para consolidar la convergencia regional. Una estrategia multilateral, con un fuerte liderazgo de Estados Unidos, es esencial en esta perspectiva para el progreso futuro en esta materia. ${ }^{17}$ En este sentido, el papel renovado y activo de la OEA es importante en la construcción de una comunidad de democracias vinculadas por lazos económicos y creencias políticas convergentes en el hemisferio.

La creación de una comunidad económica es otro propósito de la convergencia regional entre los liberales. Por lo tanto, los mecanismos multilaterales más promisorios en el desarrollo del comercio y en la propagación de la economía de mercado en la región son los acuerdos de libre comercio como el NAFTA. Aun cuando existe un creciente interés y entusiasmo en casi todos los países del continente por estrechar lazos económicos con los Estados Unidos, un acuerdo de libre comercio regional en los próximos años dependerá del comportamiento tanto del país del Norte como de las economías hemisféricas. ${ }^{18}$

En suma, los liberales perciben y apoyan una visión optimista de las relaciones interamericanas en la cual todos los Estados del hemisferio, con la excepción de Cuba, comparten una agenda similar. En otras palabras, la promoción de la democracia, el crecimiento económico y el mejoramiento de la agenda social son los valores y politicas fundamen-

\footnotetext{
${ }_{16}$ Abraham F. Lowenthal, "Rediscovering Latin America", op. cit., pp. 35-36.

${ }^{17}$ Robert Pastor, "The Latin American Option", op. cit., p. 112.

${ }^{18}$ Véase Peter Hakim, "NAFTA and After: A New Era for the United States and Latin America?", op. cit., pp. 100-102.
} 
tales y primarios que están definiendo una nueva era de sustantiva simetría en el hemisferio occidental. 19

\section{Comentarios finales.}

Las recientes relaciones hemisféricas han estado influenciadas por los contenidos y prescripciones de la nueva estrategia norteamericana de la ampliación. Su carácter pragmático —que combina valores como democracia y economía de mercado con los intereses geoestratégicos tradicionales - ha favorecido una política estadounidense más consensual. En efecto, existe actualmente una amplia convergencia política e intelectual en Estados Unidos respecto a las materias y valores más importantes a considerar y promover en el hemisferio.

No obstante, existen también desacuerdos entre los especialistas y actores políticos norteamericanos. Estas divergencias se manifiestan a través de distintas percepciones, explicaciones y propuestas políticas que están presentes en el nuevo rol hemisférico de Estados Unidos. De esta forma y de acuerdo a las categorías usadas anteriormente, tanto la visión realista (cautelosa) como la propuesta liberal (optimista) difieren en lo que dice relación con la prioridad que la región tiene para la política exterior norteamericana.

El énfasis en la explicación acerca del desarrollo regional y la estabilidad hemisférica —considerados ambos en términos del interés nacional estadounidense- es otra divergencia entre realistas y liberales. Estos últimos asumen al desarrollo y a la estabilidad de una manera comprehensiva y comunitaria, esto es, afirmando que la democracia significa un valor compartido para avanzar en el crecimiento económico y para reforzar la equidad social. Mientras que para los realistas, el desarrollo regional y la estabilidad hemisférica son asumidas sólo en el interés de Estados Unidos.

Finalmente, las prescripciones e instrumentos políticos también difieren entre realistas y liberales. Para los primeros existen prioridades hemisféricas relacionadas con aquellos temas que son percibidos de interés para la seguridad norteamericana. Así, para los realistas, los llamados temas intermésticos, es decir aquellos que envielven aspectos y actores domésticos e internacionales como el terrorismo, drogas, etc., requieren preferentemente un tratamiento bilateral por parte de las políticas de los respectivos Estados.

${ }^{19}$ Ver Richard Feinberg, "Substantive Symmetry in Hemispheric Relations", op. cit., p. 158. 
En cambio, para los liberales las prioridades hemisféricas están relacionadas con los valores democráticos, económicos y sociales como propósitos comunes de una agenda compartida. En cuanto a instrumentos de política exterior se refiere, esta categoria promueve la cooperación regional para abordar las materias de carácterinterméstico, especialmente a través de mecanismos multilaterales y organizaciones regionales.

En síntesis, a pesar de las oportunidades de mayor convergencia que ofrece la nueva estrategia exterior norteamericana a sus visiones tradicionales de política hemisférica, cabe consignar que persisten las diferencias entre ellas en virtud de sus propios supuestos y prioridades a partir de los cuales abordan la realidad regional e internacional. 